\title{
Eficácia de diferentes dosagens do formulado fúngico à base de Beuveria bassiana (Vuill, 1912) no controle de adultos de Hedypathes betulinus (Klug, 1825) (Coleoptera: Cerambycidae)
}

\author{
Efficacy of different dosages of the fungal formulation based on \\ Beauveria bassiana (Vuill, 1912) in the control of Hedypathes betulinus \\ (Klug, 1825) (Coleoptera: Cerambycidae) adults
}

\author{
Priscila Cristina Gomm ${ }^{[a]}$, Rui Scaramella Furiatti ${ }^{[b]}$, \\ Edemar Baranek ${ }^{[\mathrm{c}]}$, Luciane Tlumaske ${ }^{[\mathrm{d}]}$, Fernanda Osmara Wagner ${ }^{[\mathrm{e}]}$
}

[a] Engenheira agrônoma, Universidade Estadual de Ponta Grossa (UEPG), Ponta Grossa, PR - Brasil, e-mail: priscilagomm@yahoo.com.br
${ }^{[b]}$ Engenheiro agrônomo, Doutor em Ciências Biológicas (Entomologia), professor do Departamento de Fitotecnia e de Fitossanidade,
Universidade Estadual de Ponta Grossa (UEPG), Ponta Grossa, PR - Brasil, e-mail: furiatti@myzus.com.br
[c] Engenheiro agrônomo, Universidade Estadual de Ponta Grossa (UEPG), Ponta Grossa, PR - Brasil, e-mail: edemarjb@yahoo.com.br
[d] Engenheira agrônoma, Universidade Estadual de Ponta Grossa (UEPG), Ponta Grossa, PR - Brasil, e-mail: lutlu@hotmail.com
${ }^{[e]}$ Engenheira agrônoma, Universidade Estadual de Ponta Grossa (UEPG), Ponta Grossa, PR - Brasil, e-mail: nanda_agro@hotmail.com

\section{Resumo}

A erva-mate (Ilex paraguariensis St. Hil.) é uma cultura de importância econômica para a região sul do Brasil, podendo ser explorada na forma nativa ou cultivada. Em áreas de monocultivo, essa espécie apresenta maiores problemas com pragas. A broca-da-erva-mate Hedypathes betulinus (Klug, 1825) é a principal praga da cultura. Com o objetivo de avaliar a porcentagem e o tempo de mortalidade de adultos de $H$. betulinus, utilizou-se o fungo entomopatogênico Beawveria bassiana (Vuill, 1912), cepa CG 716. O delineamento experimental foi em blocos casualizados com seis tratamentos e quatro repetições. Os tratamentos foram: 0; 2; 26; 50 ; 74 e 98 $\mathrm{ml}$ da formulação à base de B. bassiana (Bovemax) com 107 esporos/ml. Foram liberados 20 adultos de $H$. betulinus no centro de cada parcela. As dosagens foram diluídas em dez litros de água, os quais foram aplicados no tronco da erveira. Após sete dias foram coletados todos os insetos encontrados na área do ensaio, os quais foram transportados ao laboratório e individualizados em copos plásticos contendo ramos de erva-mate como alimento. Os insetos foram avaliados por um período de 60 dias após a aplicação do fungo, sendo que os insetos mortos foram retirados e levados individualmente à câmara úmida, objetivando verificar a extrusão do fungo. Os resultados foram submetidos à análise de variância e as diferenças entre as médias, quando significativas, foram comparadas pelo teste de Tukey a 5\%. O tempo médio que o fungo levou para matar o inseto foi de 34,05 dias. A eficácia observada não foi significativamente proporcional à elevação nas dosagens do formulado. As dosagens de $50 \mathrm{ml}$ e $98 \mathrm{ml}$ foram semelhantes entre si e também as únicas que diferiram da testemunha, sendo que o tratamento com $50 \mathrm{ml}$ apresentou eficácia de 33,26\%.

Palavras-chave: Erva-mate. Fungo entomopatogênico. Broca-da-erva-mate. 


\section{Abstract}

Erva-mate (Ilex paraguariensis St. Hil.) is an economically important culture for Brazilian South region, being possible to exploit it native or cultivated. In monoculture areas, these species presents more problems with pests. Broca-da-erva-mate Hedypathes betulinus (Klug, 1825) is the most common pest in the culture. Aiming to evaluate the percentage and the time of $\mathrm{H}$. betulinus adults mortality, it was used the entomopathogenic fungus Beauveria bassiana (Vuill, 1912), cepa CG 716. The experimental delineation was in blocks, with 6 treatments and 4 recurrences. The treatments were: $0 ; 2 ; 26 ; 50 ; 74$ and $98 \mathrm{ml}$ of the formulation based on B. bassiana (Bovemax) with 107spores.ml-1. It has been liberated 20 adults of $\mathrm{H}$. betulinus in the center of each parcel. Dosages were diluted in ten liters of water, which were applied in the herbal stump. After seven days, all the insects found in the study area were collected and taken to the laboratory, where they were put individually in plastic glasses with erva-mate (Ilex paraguariensis St. Hil) as food. The insects were evaluated during 60 days after the fungus application; died insects were removed and taken to a humid chamber individually, to verify the fungus extrusion. The results were undertaken to variance analyze besides the differences among the averages, when significant, were compared through the Tukey test in $5 \%$. The average time that the fungus took to kill the insect was 34.05 days. The observed efficacy was not significantly proportional to the elevation of the dosages of the formula. The dosages of $50 \mathrm{ml}$ and $98 \mathrm{ml}$ were similar to each other and also the only ones which were different of the bystander, though $50 \mathrm{ml}$ has shown 33,26\% of efficacy.

Keywords: Erva-mate. Entomopathogenic fungus. Broca-da-erva-mate.

\section{Introdução}

A erva-mate (Ilex paraguariensis St. Hil.) é uma cultura de importância econômica para a região sul do Brasil, abrangendo cerca de 180 mil propriedades dos Estados do Paraná, Rio Grande do Sul, Santa Catarina e Mato Grosso do Sul. As propriedades em que ela é cultivada são, na maioria, pequenas e médias, o que lhe assegura uma importância social expressiva (MEDRADO, 2005).

A erva-mate pode ser explorada na forma nativa ou cultivada. No sistema de monocultivo, a planta fica mais suscetível a estresses fisiológicos e, em consequência, passa a ter maiores problemas com ataques de pragas e doenças.

A principal praga da erva-mate é Hedypathes betulinus (Klug, 1825) (Coleoptera: Cerambycidae), um besouro do grupo dos serra-paus conhecido popularmente como Corintiano ou Broca-da-erva-mate. Os maiores danos são ocasionados pelas larvas, que durante a alimentação constroem galerias, dificultando a circulação da seiva. Isso debilita a planta, diminuindo sua produção. Se o broqueamento é muito intenso, ou se ocorrem sucessivas gerações da praga, os galhos da planta podem secar e, muitas vezes, ocorre a morte da erveira (PENTEADO; IEDE, 2005).

Os sintomas de ataque são presença de serragem próxima ao colo das erveiras eplantas fracas com folhagem escassa e amarelada. Em plantios nos quais o ataque é intenso, encontra-se grande número de galhos quebrados, em razão do enfraquecimento pelas galerias e pela ação do vento. Os galhos se quebram justamente na altura das galerias anelares, praticadas pelas larvas, que geralmente permanecem no galho quebrado (CASSANELLO, 1993).

Atualmente, o método de controle mecânico, pela catação dos adultos de $H$. betulinus, é o mais utilizado. Apesar de primitivo, esse recurso é adotado pela maioria dos produtores em função da sua eficiência e fácil execução, principalmente quando há disponibilidade de mão de obra (LEITE et al., 2006).

Nessa cultura não é permitida a aplicação de nenhuma classe de moléculas inseticidas. Aliado a isso, o fato do produto ser consumido in natura reforça a necessidade de medidas de controle inseridas em um programa de Manejo Integrado de Pragas, visando a um produto final sem resíduos tóxicos (BORGES, 2007). Nesse contexto, a utilização de fungos entomopatogênicos surge como uma alternativa de controle, pois muitos deles apresentam baixo impacto ambiental.

A ocorrência natural do fungo Beauveria bassiana (Bals.) Vuill, infectando adultos de H. betulinus, abre perspectivas para sua utilização visando a assegurar a melhoria fitossanitária e, consequentemente a qualidade 
e produtividade da erva-mate, atendendo às atuais exigências do mercado (BORGES, 2007). A aplicação de formulados é uma alternativa para melhorar o índice de controle, pois a ocorrência natural do fungo é baixa.

Neste trabalho utilizou-se um formulado proveniente da cepa CG 716, isolada por Leite et al. (2000), por apresentar melhor eficácia, se comparada às demais. O objetivo deste artigo foi avaliar a porcentagem e o tempo de mortalidade de adultos de $H$. betulinus expostos a diferentes dosagens de B. bassiana formulados em óleo emulsionável (Bovemax).

\section{Material e métodos}

O experimento foi instalado no dia 15 de fevereiro de 2008, no erval da ervateira Bitumirim, na fazenda Vila Nova, localizada no município de Ivaí, PR. O delineamento experimental foi em blocos casualizados, com seis tratamentos e quatro repetições. A divisão dos tratamentos dentro do bloco e entre blocos foi feita utilizando-se fitas coloridas (cada cor representando um tratamento), que eram amarradas na bordadura da parcela. Cada parcela continha 81 árvores, entre as quais foram marcadas as 10 centrais. Estas foram marcadas para a liberação dos insetos, previamente identificados com diferentes colorações no tórax, indicando o respectivo tratamento.

Foram liberados 20 insetos nas árvores centrais da parcela ( 80 insetos/tratamento), perfazendo um total de 480 insetos liberados em todos os tratamentos. Esses insetos foram coletados em áreas vizinhas da fazenda no mesmo dia da instalação do experimento.

O formulado em óleo emulsinável à base de $B$. bassiana na concentração de $10^{7}$ esporos.ml ${ }^{-1}$ foi testado nas seguintes dosagens: 0, 2, 26, 50, 74, $98 \mathrm{ml}$, diluídos em dez litros de água. A concentração de esporos $\mathrm{ml}^{-1}$ utilizada na pesquisa seguiu a recomendação de Leite et al. (2003a), que concluíram que aquela é eficaz no controle de $H$. betulinus, sob condições de campo.

Após a liberação dos insetos, os tratamentos foram aplicados no tronco da erveira, com o auxilio de um pulverizador costal de dez litros, equipado com bico hidráulico e ponta jato cônico vazio, utilizando-se $150 \mathrm{ml}$ de calda/planta, em todas as plantas da parcela.

Essa metodologia tem como objetivo não deixar resíduo do fungo nas folhas (que são consumidas in natura), restringir a aplicação ao local onde os insetos adultos de $H$. betulinus caminham e ovipositam e evitar a infecção de insetos associados às outras partes da erveira, principalmente os inimigos naturais (BORGES, 2007).

Os insetos permaneceram somente seis dias em contato com as diferentes dosagens do fungo, pois períodos maiores de exposição poderiam ocasionar sua migração, dificultando as análises. Após esse período foram coletados todos os insetos encontrados nas árvores, identificados e não identificados, perfazendo um total de 571 indivíduos. Após a coleta, os insetos foram transportados para o Laboratório de Entomologia Aplicada pertencente à Universidade Estadual de Ponta Grossa, PR, onde foram individualizados em copos plásticos, medindo $11 \times 8 \mathrm{~cm}$, com tampa furada, contendo ramos de erva-mate como alimento.

Os insetos foram avaliados por um período de 60 dias após a aplicação do fungo, nos quais permaneceram em temperatura ambiente, com média de $22,8^{\circ} \mathrm{C}$ e umidade relativa média de $68,8 \%$. Observações diárias foram realizadas e os insetos mortos foram retirados e levados individualmente à câmara úmida, objetivando verificar a extrusão do fungo.

Para análise dos resultados utilizou-se os insetos marcados e os não marcados, e foram considerados somente os insetos mortos por B. bassiana, sendo desconsiderados os que morreram por causas naturais ou desconhecidas, nos tratamentos e também na testemunha. Os insetos que pertenciam a um tratamento de menor concentração de esporos e que no momento da coleta encontravam-se em tratamentos com dosagens maiores foram enquadrados no de maior concentração.

A eficácia foi avaliada mediante a fórmula de Henderson e Tilton, dada pela fórmula abaixo:

$\%$ de eficácia $=1-(\mathrm{Td} / \mathrm{Ta} \times \mathrm{Ca} / \mathrm{Cd}) \times 100$

Onde:

Ta = número de indivíduos vivos antes do tratamento;

$\mathrm{Td}=$ indivíduos vivos depois do tratamento; 
$\mathrm{Ca}=$ número de indivíduos vivos na testemunha antes do tratamento;

$\mathrm{Cd}=$ número de indivíduos vivos na testemunha depois do tratamento.

Os dados foram submetidos à análise de variância (ANOVA) e a diferença entre as médias, quando significativa, foi comparada pelo teste de Tukey a 5\% de probabilidade.

\section{Resultados e discussão}

A coleta dos insetos, feita seis dias após a aplicação dos esporos do fungo, revelou que a população de H. betulinus nas parcelas do experimento era desuniforme (Tabela 1), o que justifica o uso da fórmula de Henderson e Tilton, que considera a população inicial dos tratamentos e da testemunha, na estimativa da eficácia relativa.

As condições favoráveis medidas durante o período no qual os insetos ficaram expostos ao fungo no campo podem ser consideradas próximas das ideais, conforme Alves (1986), com umidade relativa em torno de $90 \%$ e temperatura na faixa de $23^{\circ} \mathrm{C}$ a $28^{\circ} \mathrm{C}$.

Tabela 1 - Coleta incial, número de indivíduos, porcentagem média de adultos de Hedypathes betulinus vivos e a eficácia de Beauveria bassiana (cepa CG 716), após os 60 dias do tratamento em erva mate. Ivaí, Paraná, 2008.

\begin{tabular}{lccc}
\hline \multirow{2}{*}{$\begin{array}{l}\text { Tratamento } \\
\text { (ml/ 10 1 de água do } \\
\text { formulado) }\end{array}$} & $\begin{array}{l}\text { Média população inicial } \\
\text { (coleta) }\end{array}$ & \multicolumn{2}{c}{ Após 60 dias do tratamento } \\
\cline { 3 - 4 } & $27,0 \mathrm{ab}$ & Média (\% insetos vivos) & Eficácia (\%) \\
\hline $98 \mathrm{ml}$ & $27,5 \mathrm{ab}$ & $67,6 \mathrm{~b}$ & 31,43 \\
$74 \mathrm{ml}$ & $28,5 \mathrm{a}$ & $78,4 \mathrm{ab}$ & 22,53 \\
$50 \mathrm{ml}$ & $24,2 \mathrm{ab}$ & $66,3 \mathrm{~b}$ & 33,26 \\
$26 \mathrm{ml}$ & $18,0 \mathrm{ab}$ & $81,8 \mathrm{ab}$ & 17,37 \\
$2 \mathrm{ml}$ & $17,5 \mathrm{~b}$ & $77,3 \mathrm{ab}$ & 21,09 \\
Testemunha & 20,20 & $98,9 \mathrm{a}$ & - \\
$\mathrm{CV}(\%)$ & 4,14 & 15,64 & \\
F & 0,01 & 3,69 & \\
P & 10,81 & 0,01 & \\
DMS & 27,58 & \\
\hline
\end{tabular}

Nota: Médias seguidas de mesma letra não diferem estatisticamente entre si pelo teste de Tukey a $5 \%$ de probabilidade

Decorridos os 60 dias da aplicação dos esporos de B. bassiana, observou-se que os tratamentos nas dosagens de $50 \mathrm{ml}$ e $98 \mathrm{ml}$ reduziram significativamente a população do inseto, em relação à testemunha (Tabela 1). O tempo médio que o fungo levou para matar o inseto foi de 34,05 dias, considerando o dia da aplicação como data de partida, o que está de acordo com Leite et al. (2003b), os quais observaram que o tempo médio foi de 30,6 dias. Depois da morte dos insetos, a extrusão do fungo ocorreu, em média, após três dias.

A eficácia observada não foi significativamente proporcional à elevação na dosagem do formulado fúngico, tendo a eficácia dos tratamentos permanecida semelhante entre si. Esse fato pode estar ligado à migração de insetos entre as parcelas e a fatores ainda desconhecidos.

Considerando o ciclo do fungo após a infecção estar efetivada (ao redor de 30 dias), pode-se conjeturar que a mortalidade não seria maior em avaliações futuras no laboratório, além dos 60 dias. Portanto, durante os seis dias em que os insetos ficaram em contato com o fungo, obteve-se um controle da população de $33,26 \%$ na dosagem mais eficiente. 
Entretanto, se tivéssemos deixado os insetos por um período de exposição maior ao fungo, possivelmente o índice de eficácia aumentaria proporcionalmente. A extensão no tempo de exposição ao fungo não foi efetuada graças ao exposto anteriormente na metodologia.

Leite et al. (2006) verificaram a persistência dessa mesma cepa e obtiveram índices altos de infecção e uma redução de $50 \%$ da população de $H$. betulinus, após dois meses da aplicação. A longevidade desses esporos também foi estudada por Borges (2007), a qual alcançou 120 dias após a aplicação no campo. Segundo esse autor, um fator que contribui para tal persistência é o fato do fungo ser formulado à base de óleo, o qual auxilia a sobrevivência do conídio e sua aderência à cutícula do inseto.

Outro fatorimportante na epizootia do fungoé a contaminação entre os insetos no acasalamento. Borges (2007) constatou que existe uma alta mortalidade de machos que entram em contato com fêmeas contaminadas.

Quando infectados, os insetos procuram os lugares mais altos das plantas, possivelmente objetivando elevar a temperatura corpórea e, com isso, matar o fungo. Esse fenômeno contribui também na eficácia do fungo, multiplicando seus esporos e a sua disseminação na planta, elevando o potencial de controle dos insetos presentes (BORGES, 2007).

É importante salientar a importância do controle biológico, o qual não elimina a população da praga, mas objetiva reduzi-la de modo que não cause danos econômicos. Assim, permite a ocorrência de outros agentes de controle como predadores, parasitóides e até mesmo do próprio fungo, que pode permanecer ativo indefinidamente na área.

\section{Conclusão}

O formulado fúngico à base de B. bassiana na dosagem de $50 \mathrm{ml} \mathrm{em} \mathrm{dez} \mathrm{litros} \mathrm{de} \mathrm{água} \mathrm{foi} \mathrm{a} \mathrm{mais}$ eficaz, resultando em 33,26\% de controle de $H$. betulinus, durante seis dias de exposição, matando o inseto em um tempo médio de 34,05 dias.

\section{Referências}

ALVES, S. B. Controle microbiano de insetos. São Paulo: Manole, 1986.

BORGES, L. R. Eficiência de Beauveria bassiana (BALS.) VUILL. (Deuteromycota) para o controle de Hedypathes betulinus (KLUG) (Coleoptera: Cerambycidae) em erva-mate, Ilex paraguariensis ST. HIL. (Aquifoliaceae). 2007.102 f. Tese (Doutorado Ciências Biológicas) - Universidade Federal do Paraná, Curitiba, 2007.

CASSANELLO, A. M. L. Ciclo de vida e aspectos morfológicos de Hedypathes betulinus (Klug, 1825) (Coleoptera, Cerambycidae, Lamiinae), broca-da-erva-mate (Ilex paraguariensis St. Hil.). 1993. 59 f. Dissertação (Mestrado em Entomologia) - Curso de Pós-graduação em Ciências Biológicas, Universidade Federal do Paraná, Curitiba, 1993.

LEITE, M. S. P. et al. Seleção de linhagens de fungos entomopatogênicos para o controle de Hedypathes betulinus (Klug, 1895) (Coleoptera: Cerambycidae) em laboratório e eficiência da linhagem selecionada em campo. In: CONGRESSO SUL-AMERICANO DA ERVA-MATE, 2., REUNIÃO TÉCNICA DA ERVA-MATE, 3., 2000, Encantado. Anais... Encantado, RS: Congresso Sul-Americano de Erva-Mate, 2000. p. 27-37.

LEITE, M. S. P.; IEDE, E. T.; PENTEADO, S. R. C. Manejo integrado de pragas em erva-mate para o controle da broca-da-erva-mate, com ênfase ao uso de entomopatógenos. In: CONGRESSO SUDAMERICANO DE LA YERBA MATE, 4., 2006. Posadas, Argentina. Anais... Posadas, Argentina: Congresso Sudamericano de la Yerba Mate, 2006. CD-ROM.

LEITE, M. S. P.; PENTEADO, S. R. C.; OLIVEIRA, S. Avaliação de duas espécies de fungos entomopatogênicos para o controle de Hedypathes betulinus (Klug, 1825) (Coleoptera: Cerambycidae) em laboratório. In: CONGRESSO SUL-AMERICANO DA ERVA-MATE 3., 2003, Chapecó. Anais... Chapecó: Congresso Sul-Americano de Erva-Mate, 2003a. p. 16-19. 
. Eficiência do fungo Beauveria bassiana (Bals.) Vuill. no controle de Hedypathes betulinus (Klug, 1825) (Coleoptera: Cerambycidae) em campo, In: CONGRESSO SUL-AMERICANO DA ERVA-MATE 3., 2003, Chapecó. Anais... Chapecó: Congresso Sul-Americano de Erva-Mate, 2003b. CD-ROM.

LEITE, M. S. P. et al. Eficiência de Beauveria bassiana (BALS) Vuill formulado em óleo no controle de Hedypathes betulinus (Klug) (Coleoptera: Cerambycidae) em campo. In: CONGRESSO SUDAMERICANO DE LA YERBA MATE, 4., 2006. Posadas, Argentina. Anais... Posadas, Argentina: Congresso Sudamericano de la Yerba Mate, 2006. p. 269-272.

MEDRADO, M. J. S. Cultivo da erva-mate. 2005. Disponível em: < http://sistemasdeproducao.cnptia. embrapa.br/FontesHTML/Erva-mate/CultivodaErvaMate/apresentacao.htm>. Acesso em: 23 mar. 2008.

PAGliOSA, M. M. R.; SANTOS, H. R.; DIODATO, M. A. Patogenicidade do fungo entomopatogênico Beauveria bassiana (BALS) VUILL., em Hedypathes betulinus (KLUG, 1825), praga da erva-mate, Ilexparaguariensis St. Hil. Agrárias, v. 13 n. 1-2, p. 229-231, 1994.

PENTEADO, S. do R. C.; IEDE, E. T. Cultivo da erva-mate: pragas. 2005. Disponível em: <http:// sistemasdeproducao.cnptia.embrapa.br/FontesHTML/Erva-mate/CultivodaErvaMate/apresentacao.htm>. Acesso em: 2 abril 2008.

Recebido: 25/05/2009

Received: 05/25/2009

Aprovado: 22/12/2009

Approved: $12 / 22 / 2009$ 